\title{
Childhood-Onset Epileptic Encephalopathy Associated With Isolated Focal Cortical Dysplasia and a Novel TSCI Germline Mutation
}

Clinical EEG and Neuroscience 2018, Vol. 49(3) 187-191 (C) EEG and Clinical Neuroscience Society (ECNS) 2017

Reprints and permissions: sagepub.com/journalsPermissions.nav DOI: 10.1 I77/I5500594|769784| journals.sagepub.com/home/eeg (\$SAGE

\author{
Hannes Hoelz', Eva Coppenrath ${ }^{2}$, Konstanze Hoertnagel $^{3}$, Timo Roser', \\ Moritz Tacke', Lucia Gerstl', and Ingo Borggraefe ${ }^{1,4}$
}

\begin{abstract}
Tuberous sclerosis complex (TSC) is an autosomal-dominant inheritable neurocutaneous disease due to mutations within the TSCI and TSC2 genes. Many patients present with West syndrome, a severe epilepsy syndrome characterized by the triad of infantile spasms, an interictal electroencephalogram (EEG) pattern termed hypsarrhythmia (continuous slow activity with an amplitude higher than $300 \mu \mathrm{V}$ and multiregional spikes/polyspikes/sharp waves) and developmental regression. In this study, we report on a previously healthy patient with positive family history of epilepsy with new-onset epileptic encephalopathy at the age of 9 years. Clinical signs alone were not sufficient to establish the diagnosis of TSC but epilepsy panel screening revealed a novel frameshift mutation (c.90delA; p.Glu3 IArgfs*12) within the TSCI gene. Segregation gene analysis detected the same mutation in the mother. Cranial magnetic resonance imaging (MRI) studies from the index patient and his mother revealed a similar pattern of isolated subcortical white matter lesions resembling most likely focal cortical dysplasia (FCD) type Ilb. In summary, in these 2 related patients, a novel TSCI frameshift mutation was associated with an isolated FCD type llb in the absence of further CNS abnormalities usually encountered in patients with TSC, fostering our understanding of the broad mutation spectra in the TSCI gene and the close relationship between cortical tubers and FCD type Ilb.
\end{abstract}

\section{Keywords}

tuberous sclerosis complex, frameshift mutation, tuberous sclerosis complex I protein, epilepsy, malformations of cortical development

Received September 9, 2016; revised November 28, 2016; accepted February 5, 2017.

\section{Introduction}

Tuberous sclerosis complex (TSC) is an autosomal dominant inherited disease that occurs in a total population prevalence of 1:12500 individuals. ${ }^{1}$ Eighty percent to $90 \%$ of people with TSC are affected by epilepsy. ${ }^{2}$ Epilepsy manifests in early childhood with epileptic spasms in up to $30 \%$ of the cases though later onset with manifestations of epileptic encephalopathy have also been reported. ${ }^{3}$ The structural hallmarks of central nervous system (CNS) involvement are cortical tubers resembling histologically focal cortical dysplasia with balloon cells (type IIb dysplasia), subependymal nodules and giant cell astrocytoma. ${ }^{4}$ The causative mutations for TSC are located in either TSC1 (encoding hamartin), at 9p34, and TSC2 (encoding tuberin) at 16p13.3, which are sufficient mammalian Targets of Rapamycin (mTOR)-inhibitors. ${ }^{5,6}$ In $80 \%$ of all individuals with TSC, causative mutations in these 2 genes can be detected. ${ }^{4}$ Sixty percent of these mutations occur spontaneously. Haploinsufficiency of mTOR is most likely responsible for uncontrolled cell proliferation leading to the majority of clinical symptoms in this syndrome. Of interest, mTOR inhibitors have shown to reduce seizure burden in some epileptic patients beside of reducing the size of subependymal giant cell astrocytoma (SEGA). ${ }^{7}$ Here, a novel mutation in the TSCl gene was detected, associated with an isolated brain lesions most likely resembling FCD type IIb and childhood onset epileptic encephalopathy.

\footnotetext{
'Department of Pediatric Neurology, Developmental Medicine and Social Pediatrics, Dr. von Haunersches Children's Hospital, Ludwig-MaximiliansUniversity, Munich, Germany

${ }^{2}$ Institute for Diagnostic and Clinical Radiology, Ludwig-MaximiliansUniversity, Munich, Germany

${ }^{3} \mathrm{CeGaT} \mathrm{GmbH}$, Tübingen, Germany

${ }^{4}$ Epilepsy Center (Pediatric Section), Ludwig-Maximilians-University, Munich, Germany
}

Corresponding Author:

Ingo Borggraefe, Department of Pediatric Neurology, Developmental Medicine and Social Pediatrics, Epilepsy Center, Dr. von Haunersches Children's Hospital, Ludwig-Maximilians-University, Lindwurmstraße 4, 80337 Munich, Germany.

Email: ingo.borggraefe@med.uni-muenchen.de 


\section{Patient and Methods}

The index patient was admitted the hospital (Department of Pediatric Neurology of the University of Munich) because of recurrent seizures. Publication of patient data was authorized by the patient and its legal representative. Informed written consent was obtained from the parents prior to genetic analysis as required by German law.

\section{EEG and MRI}

Repeated 24-channel electroencephalography (EEG) recordings using Xltek hardware and software equipment (Natus DBA, Excel-Tech Corp, Oakville, Ontario, Canada) were performed using standard adjustments $(0.5 \mathrm{~Hz}$ low-frequency filter, $70 \mathrm{~Hz}$ high-frequency filter, resistance $5-10 \mathrm{kohm}$ ). MRI of the brain was obtained using a 3-T high-resolution scanner (1.0-1.5 mm slices, $\mathrm{T} 1$ with and without gadolinium contrast enhancement, T2, and fluid attenuated inversion recovery [FLAIR] techniques) in axial, sagittal, and coronal planes (Philips Ingenia, Amsterdam, Netherlands).

\section{Genetic Analysis}

Five milliliters of ethylenediaminetetraacetic acid (EDTA) blood was drawn from the index patient and an epilepsy gene panel screening, including 77 genes for epileptic encephalopathy was performed as reported elsewhere. ${ }^{8}$ A segregation analysis was performed with $5 \mathrm{~mL}$ EDTA blood from his mother, in which the relevant area of the TSC1 gene (NM_000368.4) was amplified by polymerase chain reaction (PCR). By using flanking and internal primers bidirectionally direct sequencing was performed (96 capillary sequencing tool 3730x1 DNA Analyzer from Applied Biosystems, Carlsbad, CA, USA). Interpretation of genetic results was in accordance with the recently updated American College of Medical Genetics guidelines for the interpretation of sequence variants. ${ }^{9}$

\section{Results}

\section{Patient History and Clinical Findings}

The index patient is a 9-year-old male, who received medical attention because of several epileptic seizures per day. The semiology of seizures changed over time, from initially solely dialeptic seizures to generalized tonic-clonic, automotor, astatic, and myoclonic seizures. On physical examination, dental enamel pits, 2 hypomelanotic macules on his back and neck, and ubiquitous disseminated confetti skin lesions were present. Ultrasound sonography was unremarkable with no signs of cardiac rhabdomyomas or renal angiomyolipomas. At that point he did not fulfill the clinical diagnostic criteria of TSC. ${ }^{10}$ The 41-year-old mother was diagnosed with epilepsy of unknown etiology at the age of 23 years and has been treated successfully with oxcarbazepine (transient discontinuation of treatment was followed by recurrence of generalized tonic-clonic seizures).
The father and 2 sisters of the index patient are healthy. Ethosuximid, Clobazam, and Levetiracetam (LEV) in the index patient did not prove successful. Introduction of oxcarbazepine (in combination with LEV) stopped seizure recurrence. In addition, EEG abnormalities (both encephalopathic and epileptic patterns) resolved completely (Figure 1b).

\section{EEG and Neuroimaging}

Repeated EEG recordings revealed lack of normal background activity, continuous generalized slowing, and epileptic discharges mostly consisting of generalized slow spike wave complexes (Figure 1a). In addition, paroxysmal fast activity was recorded. The cranial MRI of the index patient showed an isolated subcortical FLAIR-hyperintense wedged-shaped lesion within the right inferior frontal gyrus (Figure 2a and b) without contrast enhancement suitable for fulfilling MRI criteria for both cortical tubers and focal cortical dysplasia (FCD) type IIb. A similar shaped isolated lesion was found in the mother located within the cingulate gyrus (Figure $2 \mathrm{~d}$ and e). The right cingulate gyrus of the index patient as well as the right inferior frontal gyrus of the mother was not affected (Figure 2c and f). There was no evidence for multiple cortical tubers, subependymal nodules, or subependymal giant cell astrocytomas in both the index patient and his mother.

\section{Mutation Analysis}

Genetic testing of the index patient revealed a previously undescribed heterozygous frameshift mutation (c.90delA; p.Glu31Argfs*12) within the TSC1 gene, which was also detected in the affected mother. There is no reported allele frequency of this mutation within the Exom Aggregation Consortium (ExAC) database. Application of criteria of the American College of Medical Genetics was sufficient to classify the mutation as pathogenic.

\section{Discussion}

We report on 2 related patients with the same TSC1 mutation and isolated FCD type IIb in the absence of further brain abnormalities usually encountered in TSC. Both the index patient and his mother are heterozygous for a mutation (c.90delA; p.Glu31Argfs*12) in the TSC1 gene. This frameshift mutation leads to an untimely stop codon causing either a truncated protein or a nonsense-mediated mRNA decay. Most likely these alterations cause dysregulation of mTOR resulting in uncontrolled cell growth. The mutation is not listed in the single nucleotide polymorphism database (dbSNP), the human gene mutation database and the ExAC database. Of interest, a frameshift mutation (p.Ser30GlnfsTer21) in close vicinity to the mutation reported in this study was reported once in the ExAC database in an unaffected individual. One may speculate whether this individual was unaffected or revealed similar mild clinical pictures and/or FCD IIb on MRI, which came not to medical attention 


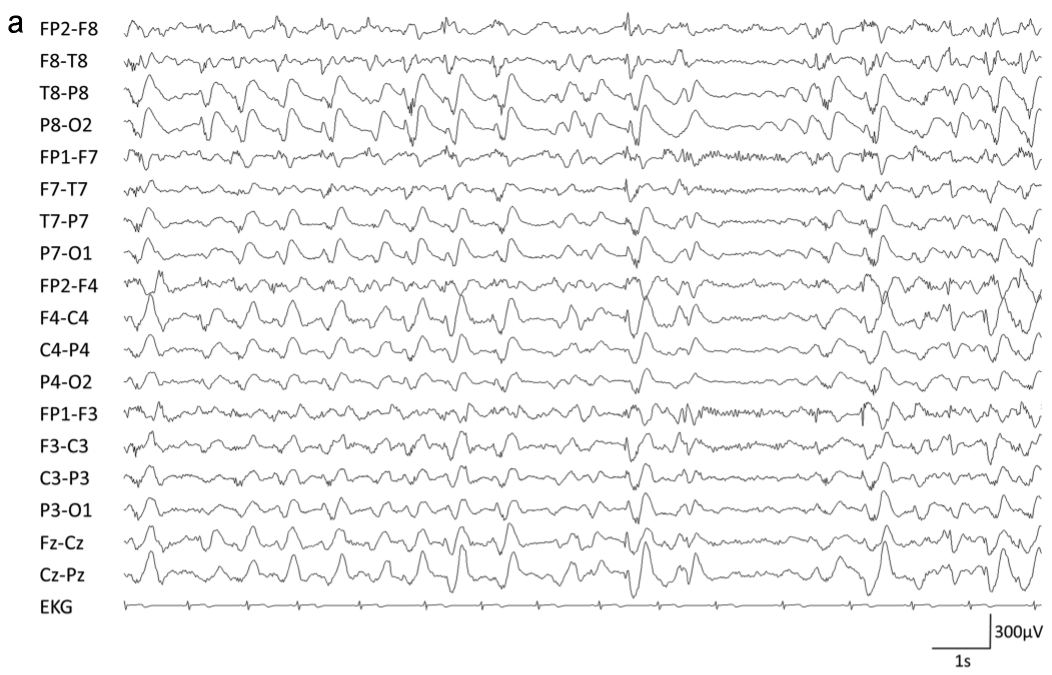

b

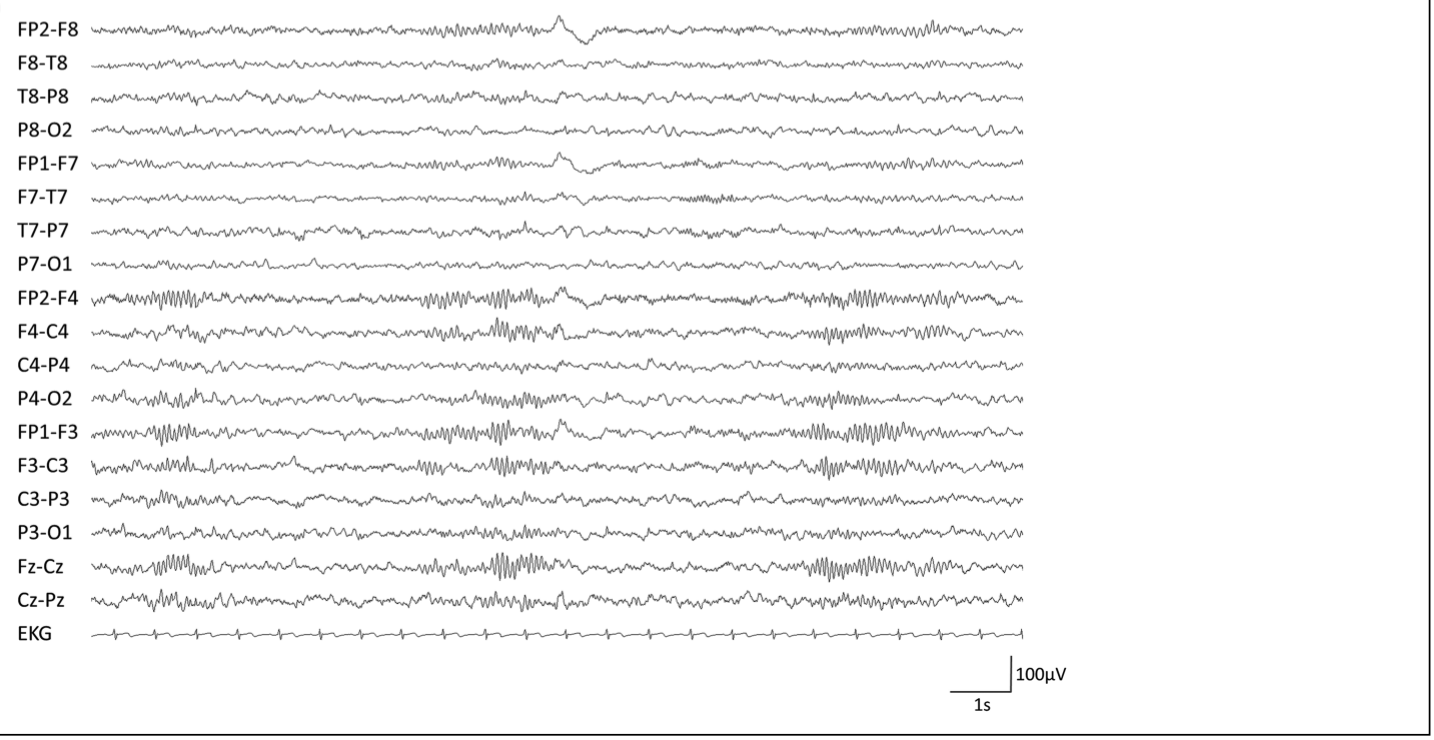

Figure I. Sleep-EEG of the index patient at onset of seizures reveals lack of normal background activity and lack of normal sleep elements. Continuous generalized slowing and epileptic discharges mostly consisting of generalized slow spikes wave discharges are present (a). SleepEEG after introduction of oxcarbazepine at $20 \mathrm{mg} / \mathrm{kg}$ reveals normal sleep elements (sleep spindle and k-complex); no epileptic discharges were recorded (b).

due to the absence of seizures. However, the mutation spectra of the TSC genes are very heterogeneous, making a conclusive genotype to phenotype correlation difficult. ${ }^{11}$

The MRI findings in our patients indicate FCD type IIb, which is a major cause of antiepileptic drug-resistant epilepsy. ${ }^{12}$ Overactivation of mTOR has been shown to be pathologically relevant in both TSC and FCD type IIb, which suggests a mechanistic link between these lesions. TSC1 gene mutations can be detected within surgical specimens of FCD type IIb patients collected during resective epilepsy surgery. ${ }^{13,14}$ However, while the occurrence of somatic brain mosaics of TSC1 mutations are established in FCD IIb, this case study confirmed the association of a TSC1 germline mutation and isolated focal cortical dysplasia. Typical clinical TSC criteria might be infrequent and not sufficient to fulfill the criteria to make the diagnosis alone. ${ }^{15}$
In order to gather evidence of causality between cortical dysplasia and seizure-onset a long-term EEG video monitoring study would have been desirable in our diagnostic workup of the index patient. Hence there is no definite proof for seizure onset to be caused by cortical dysplasia in this case. The patient revealed some electroclinical features compatible with Lennox-Gastaut syndrome (LGS). LGS may be associated with TSC. In addition, diffuse epileptic encephalopathy may be due to focal brain lesions. Alternatively, one could hypothesize the presence of brain areas with disturbed mTOR regulation that are not prominent enough to be detected by even high-resolution MRI but serve as an epileptogenic zone. Another hint for the relevance of the focal brain lesion in this case is the clear response to oxcarbazepine in both patients. However, presurgical evaluation is 

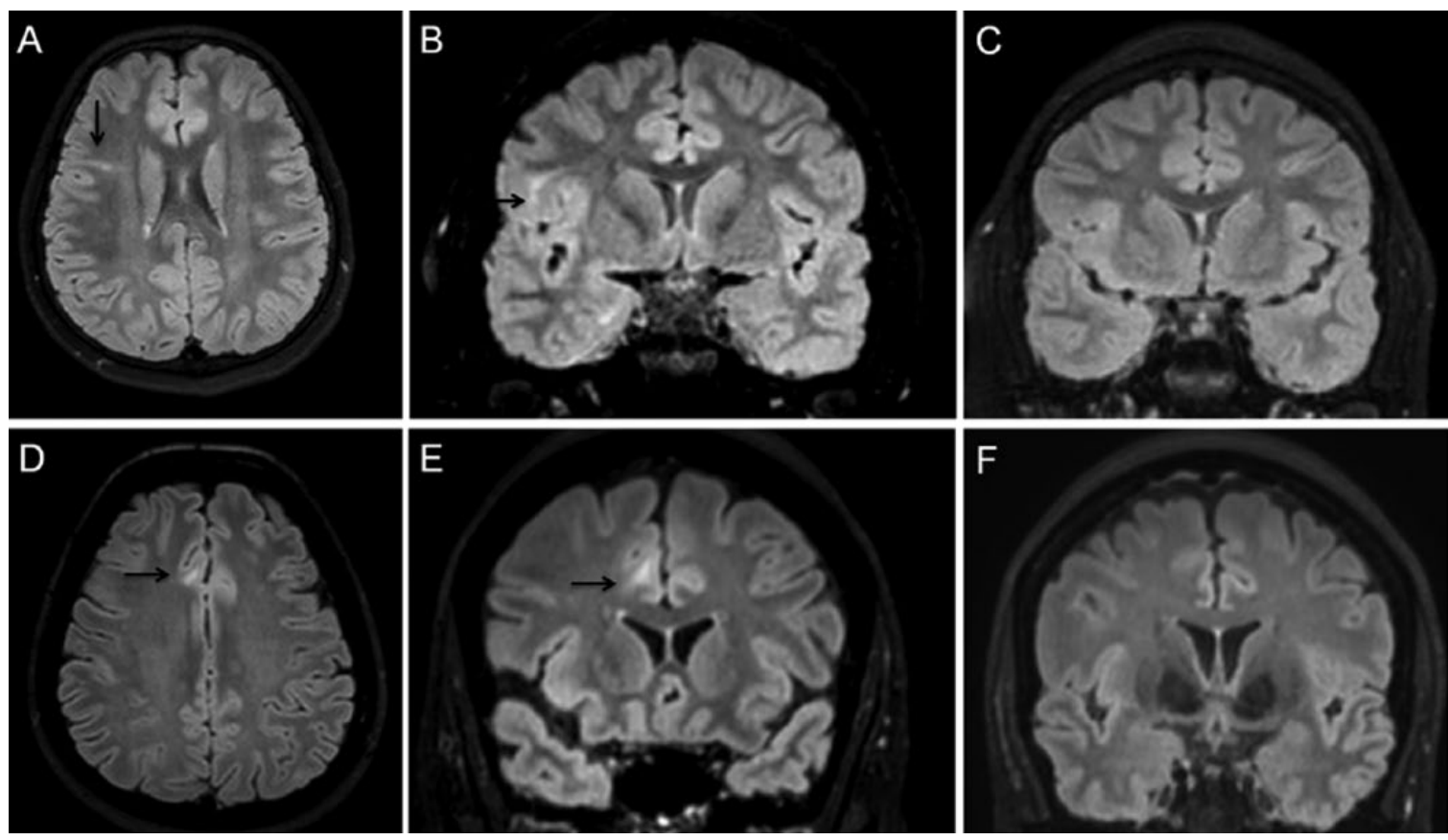

Figure 2. Cranial MRI FLAIR slices show focal wedged-shaped hyperintense lesion consistent with focal cortical dysplasia (FCD) type Ilb in both the index patient and his mother (black arrows). Panel A (axial) + B (coronal) show the index patient: lesions within the right inferior frontal gyrus. Panel D (axial) + E (coronal) show the patient's mother: lesion within the right cingulate gyrus. For comparison, Panel $C$ shows the unaffected right cingulate gyrus of the index patient and panel $F$ shows the unaffected right inferior frontal gyrus of the mother.

warranted in this patient in case of further failure of antiepileptic drug treatment.

In conclusion, we identified a novel germline mutation, c90delA; p.Glu31 Argfs*12, in the TSC1 gene in 2 related patients with FCD IIb. The presence of TSC1 germline mutations should be considered in patients with FCD IIb and concomitant criteria for TSC especially in familiar epilepsy even if the clinical signs are mild and not sufficient to define the diagnosis of TSC alone.

\section{Acknowledgments}

The authors gratefully extend their gratitude to the index patient and his mother, who are affected by TSC, for their participation and cooperation in this study.

\section{Author Contributions}

$\mathrm{HH}$ and IB drafted the manuscript. IB reviewed the manuscript and analyzed the EEG data. EC performed and analyzed the MRI data. KH performed the genetic analysis and the interpretation of genetic findings. TR, LG and MT revised the manuscript and treated the patient. All authors reviewed the manuscript.

\section{Declaration of Conflicting Interests}

The author(s) declared no conflicts of interest with respect to the research, authorship, and/or publication of this article.

\section{Funding}

The author(s) received no financial support for the research, authorship, and/or publication of this article.

\section{References}

1. O'Callaghan FJ, Shiell AW, Osborne JP, Martyn CN. Prevalence of tuberous sclerosis estimated by capture-recapture analysis. Lancet. 1998;351:1490.

2. Thiele EA. Managing and understanding epilepsy in tuberous sclerosis complex. Epilepsia. 2010;51(suppl 1):90-91.

3. Saxena A, Sampson JR. Epilepsy in tuberous sclerosis: phenotypes, mechanisms, and treatments. Semin Neurol. 2015;35: 269-276.

4. Crino PB, Nathanson KL, Henske EP. The tuberous sclerosis complex. N Engl J Med. 2006;355:1345-1356.

5. European Chromosome 16 Tuberous Sclerosis Consortium. Identification and characterization of the tuberous sclerosis gene on chromosome 16. Cell. 1993;75:1305-1315.

6. van Slegtenhorst M, de Hoogt R, Hermans C, et al. Identification of the tuberous sclerosis gene TSC1 on chromosome 9q34. Science. 1997;277:805-808.

7. Franz DN, Belousova E, Sparagana S, et al. Long-term use of everolimus in patients with tuberous sclerosis complex: final results from the EXIST-1 study. PLoS One. 2016;11: e0158476.

8. Lemke JR, Riesch E, Scheurenbrand T, et al. Targeted next generation sequencing as a diagnostic tool in epileptic disorders. Epilepsia. 2012;53:1387-1398.

9. Richards S, Aziz N, Bale S, et al. Standards and guidelines for the interpretation of sequence variants: a joint consensus recommendation of the American College of Medical Genetics and Genomics and the Association for Molecular Pathology. Genet Med. 2015;17:405-424. 
10. Northrup H, Krueger DA and International Tuberous Sclerosis Complex Consensus G. Tuberous sclerosis complex diagnostic criteria update: recommendations of the 2012 Iinternational Tuberous Sclerosis Complex Consensus Conference. Pediatr Neurol. 2013;49:243-254.

11. Curatolo P, Moavero R, Roberto D, Graziola F. Genotype/phenotype correlations in tuberous sclerosis complex. Semin Pediatr Neurol. 2015;22:259-273.

12. Aronica E, Crino PB. Epilepsy related to developmental tumors and malformations of cortical development. Neurotherapeutics. 2014;11:251-268.
13. Becker AJ, Urbach H, Scheffler B, et al. Focal cortical dysplasia of Taylor's balloon cell type: mutational analysis of the TSC1 gene indicates a pathogenic relationship to tuberous sclerosis. Ann Neurol. 2002;52:29-37.

14. Lim JS, Kim WI, Kang HC, et al. Brain somatic mutations in MTOR cause focal cortical dysplasia type II leading to intractable epilepsy. Nat Med. 2015;21:395-400.

15. Hirfanoglu T, Gupta A. Tuberous sclerosis complex with a single brain lesion on MRI mimicking focal cortical dysplasia. Pediatr Neurol. 2010;42:343-347. 